\title{
UNA INVESTIGACIÓN SOBRE HABILIDADES PARA EL APRENDIZAJE CIENTÍFICO
}

\author{
BANDIERA, M.,' DUPRÈ, F., IANNIELLO, M.G. ${ }^{2}$ y VICENTINI, M. ${ }^{2}$ \\ ' Departamento de Biología Celular y Desarrollo, Ćniversidad La Sapienza. Ple. A. Moro, 2.00185 Roma. \\ ${ }^{2}$ Departamento de Física, Universidad La Sapienza. Ple. A. Moro, 2. 00185 Roma.
}

\section{SUMMARY}

We report on the natural sciences aspects (physics and biology) of a more complete research on the prerequisitcs which university teachers expect from freshmen, and the actual capabilities of students at the end of their secondary school. This research has been done on 120 students of 15 classes in Rome (ltaly). We have tested their ability of transposition among verbal, mathematical and graphical languages, of reading non-elementary graphs, of utilizing empirical data. The conclusion is that at the end of their high school students are largely unprepared to cope with scientific studies, because of their low training to assume analytic attitudes and to operate in formalized manners.

\section{INTRODUCCIÓN}

I.a «didáctica de las ciencias» se propone actualmente y con pleno derecho como una «disciplina». Hoy, su campo de interés se extiende, por un lado, a todas las situaciones en las que se practica la enseñanza de las ciencias, desde la escucla primaria a la universidad y a la educación permanente; por otro lado, supera los contenidos específicos de las ciencias, como la biología o a la física, para abarcar los prerrequisitos léxicos, lingüísticos, lógicos y experienciales, las habiridades cognitivas y de estudio, es decir, todas aquellas habilidades que posibilitan un acercamiento más eficaz y correcto al estudio de los fenómenos naturales. Esta ampliación hacia competencias no estrictamente disciplinarias aparece como indispensable desde varios puntos de vista: baste recordar que muchas investigaciones dedicadas al estudio y comparación entre el conocimento común y el conocimiento científico destacan una sustancial diferenciá entre los modos de interpretación del hombre común (de la calle) o unovato» (Chi 1981, Hackling 1988) respecto a los del especialista (científico). De éstas y de otras investigaciones similares (Pfundt 1990), surge la existencia de una organización compleja de hábitos mentales en los sujetos examinados, que son consolidados por la práctica cotidiana y funcionales en ella, y que representan un obstáculo difícil de identificar, superar y remover en el camino de la construcción del saber científico. Fstos hábitos mentales prevalecen sobre la disposición a aplicar reglas y a asumir comportamientos diversos de aquéllos consolidados, aun cuando estos últimos no sean correctos desde el punto de vista científico. La escuela tiene la responsabilidad de promover, ejercitar y alentar esta disposición. Investigaciones realizadas en diversos ámbitos disciplinarios han demostrado que los estudiantes, al comienzo de los estudios universitarios, son más parecidos al hombre común que al especialista, aunque hay una suerte de proporcionalidad entre la duración de la experiencia escolar y, por ejemplo, el número de términos propios del lenguaje científico que utilizan, o bien, entre las soluciones de compromiso entre las concepciones espontáneas que persisten y las científicas. Es necesario tener presente que la formación científica debe entenderse como una introducción a una forma de "comunicación especial» que no puede realizarse si no se dominan habilidades básicas, ya sean generales, interdisciplinarias o cspecíficamente disciplinarias. El logro de tales habilidades debería ser un objetivo fundamental de un proyecto didáctico y encararse sistemáticamente desde los primeros años de escolaridad. De hecho, si esto se descuida, se corre el riesgo de confiar a la espontancidad aquellos 
procesos de selección de información, de organización de los conocimientos, de perfeccionamiento de los instrumentos lógicos que constituyen el bagaje personal de quien emprende estudios especializados.

Este bagaje inadecuado adquirido en la escuela podría ser la base de las insuficiencias - de las cuales se lamentan los docentes y son causa de abandonos y equivocaciones-de los estudiantes que se inscriben en la universidad: en esta fase de la instrucción es demasiado tarde para tomar medidas tendentes a una recuperación sustancial. Pero, aun cuando la situación sea ya irreversible, se podría al menos, obligar al estudiante a reflexionar sobre el estado de sus habilidades, incluso en relación con la oportunidad de elegir una carrera determinada. Así también es útil que, en esta fase, los docentes encuentren el modo de confrontar sus expectativas de «especialista» con lo que realmente el estudiante sabe y sabe hacer, con su modo de razonar.

El interés común en este tipo de problemas ha impulsado a un grupo de docentes de la Universidad La Sapienza, con competencias heterogéneas en el plano disciplinar, a proyectar y desarrollar una búsqueda dirigida a la definición de un instrumento idóneo para evaluar al estudiante respecto de un cierto número de habilidades de estudio fundamentales $y$, al mismo tiempo, apto para proporcionar a los docentes universitarios información sobre el estado de las habilidades cognitivas con las cuales los estudiantes provenientes de la escuela secundaria se presentan a la universidad

Dicha búsqueda se organizó en dos investigaciones: la primera, con los docentes universitarios, dirigida a identificar qué habilidades cognitivas, referidas a los métodos y a los contenidos, demandan los docentes en diversos cursos universitarios; la segunda, con los estudiantes de la escuela secundaria superior, dirigida a averiguar las habilidades cognitivas que poseen.

En lo referente a la investigación en los docentes, excluida toda pretensión de que sea completa, se eligió una muestra de aquéllos que enseñan en los primeros años de los cursos universitarios. A los docentes interesados les fue presentado un cuestionario en el cual se les pedía que mencionaran Ios prerrequisitos que consideraban necesarios para cursar provechosamente las asignaturas de los primeros años de las carreras universitarias: los datos recogidos se tradujeron en la definición de algunas habilidades cognitivas fundamentales, $y$ en relación con ellas se formuló un cuestionario escrito que fue propuesto posteriormente a estudiantes de los últimos cursos de cada una de las opciones de la escuela secundaria superior.

Con respecto al cuestionario pasado a los estudiantes, la investigación sobre las habilidades cognitivas fue llevada a cabo transversalmente en varios ámbitos disciplinarios (biología, física, literatura italiana, psicología, historia) (Bandiera 1990). En el párrafo dedicado a los resultados de la investigación, éstos se presentan relacionados con las cuestiones intradisciplinarias consideradas de particular interés para el estudio de las discipiinas científicas.

\section{EXPECTATIVAS DE LOS DOCENTES UNIVERSITARIOS}

La encuesta llevada a cabo entre los docentes universitarios de varios cursos de licenciatura (en el área de física y biologia) de la Universidad La Sapienza de Roma, puso en evidencia que las expectativas sobre la preparación de los alumnos inscritos en el primer año, (probablemente a causa de la desconfianza crónica en la capacidad de brindar formación científica de la escuela secundaria), no se refieren tanto a los contenidos específicos de las disciplinas como a las habilidades básicas, que podrían sintetizarse en la tradicional terna: «leer, escribir, hacer cálculos». Paradojalmente, fa situación optima esperada corresponde a la de una tabula rasa: no obstante, se espera del estudiante una especie de capacidad básica para formarse autónomamente, capacidad que, necesariamente, presupone el manejo de instrumentos lógico-formales. Las expectativas expresadas, por lo tanto, están muy lejos de ser mínimas. Por «leer, escribir, hacer cálculos"s se entiende un dominio calificado de las habilidades mencionadas, en el sentido de saber hacer una lectura crítica distinguiendo lo esencial de lo secundario o superfluo, de saber elaborar textos escritos respetando las reglas gramaticales, sintácticas y lógicas $y$, por último, de saber comprender y usar la matemática como lenguaje para expresión de contenidos conceptuales, por ejemplo, de la física.

DeI análisis de esas expectativas surge una imagen de ciencia fuertemente ligada al lenguaje que expresa sus contenidos: se trata de un lenguaje en el que figuran palabras-concepto que asumen un significado no ambiguo, en función del contexto; un lenguaje marcado por una gramática, que da las reglas en base a las cuales conectar las palabras para formar frases y que está dotado de un diccionario que permite traducir las frases de un problema a otro (Bronowski 1980).

Las expectativas en cuestión, analizadas en función de las tres habilidades básicas, esconden capacidades sofisticadas y específicas, siendo los docentes más o menos concientes de ello.

No obstante, las tres habilidades indicadas han permitido identificar un campo de investigación común a diversos contextos disciplinarios, una hipótesis de trabajo unificadora que se ha concretado, para la física y la biología (sobre las cuales se discute en este artículo), en la construcción de algunas pruebas referidas especificamente a la lectura de textos cientificos.

Cabe aclarar que la lectura de textos científicos, si por una parte requiere de un conjunto de conocimientos (en función del nivel de tratamiento) y de una capacidad de interpretación, desde el punto de vista «técnico» implica no sólo el dominio del lenguaje verbal sino también un amplio conocimiento del lenguaje matemático, en particular algebraico y gráfico. El «hacer cálculos», por lo tanto, no se refiere sólo a la aritmética sino también al álgebra elemental con que se expresan formalmente las leyes de la física y en la que los conceptos hallan un significado preciso. La habilidad de leer, interpretar ( $y$ 
construir) gráficos y tablas es, por otra parte, necesaria para la comunicación de los resultados experimentales y para comparar los datos experimentales con las tcorías.

Por todo esto, en lo que respecta al contexto de las ciencias experimentales, se decidió centrar la investigación sobre las habilidades de transposición de lenguajes (dcl lenguajc verbal al algebraico y gráfico y viceversa), y también -por extensión-sobre las habilidades para interpretar datos experimentales, formulando adecuadamente los resultados y conclusiones.

\section{PRESEN'TACIÓN DE LOS TESTS Y RE. SULTADOS}

El cuestionario propuesto a los estudiantes contenía 21 preguntas sobre varios ámbitos (psicológico, histórico, lingiústico) de las ciencias experimentales. A cada estudiante se le propuso un grupo de 8 preguntas. En cada clase se distribuyeron alternativamente grupos distintos de preguntas.

Se entrevistaron 120 estudiantes del último año de la escuela secundaria (15 clases de liceo clásico y científico, institutos técnicos y comerciales). El cuestionario fue presentado como un trabajo de investigación para el cual se pedía la colaboración de los estudiantes, que tenían una hora de tiempo para dar las respuestas de forma anónima.

A continuación mostramos los resultados del análisis de las pruebas sobre las habilidades de transposición de lenguajes y lectura de datos experimentales.

\section{Transposición de lenguajes}

Para obtener información sobre el desarollo de esta capacidad en estudiantes al término de los estudios medios superiores, se utilizaron cuatro tests, dos de ellos se refieren a la habilidad de escritura y dos a la de lectura.

\section{Del lenguaje verbal al lenguaje algebraico y gráfico: la "sinfonanza"}

En el primer test (Fig.1) se pide a los alumnos que expresen algebraicamente (a) y gráficamente (b) una afirmación verbal que corresponde al enunciado de una ley, pero que está formulada cn magnitudes inventadas, de modo que las respuestas no sean contaminadas por tos recuerdos de leyes aprendidas memorísticamente.

Las dos transposiciones solicitadas fueron analizadas por separado.

a) Transposición en fórmula. La respuesta que aparcce con más frecuencia es aquélla en que el estudiante escribe una simple igualdad

$$
\mathrm{S}=\mathrm{LG} / \mathrm{P}
$$

omitiendo indicar la proporcionalidad (lo que se puede hacer con una constante $\mathrm{K}$ que multiplica o por medio el símbolo $\alpha$ ): aunque este error no es excesivamente grave ( «en el límite del suficiente»), demuestra, por bo menos, superficialidad $y$, en algún modo, insensibilidad con respecto al concepto de proporcionalidad. Distinguiendo las respuestas en correctas (que expresan la proporcionalidad), suficientes (sólo la igualdad) y erróneas (de varios tipos), la fracción de formulaciones «suficientes» es superior a los $2 / 3$ de los estudiantes, mientras las respuestas correctas son inferiores a $1 / 5$.

Entre las formulaciones erróneas, aproximadamente un cuarto recurre en modos diversos (no correctos) a la escritura de proporciones.

Figura 1

Test I

En la siguiente pregunla las palabras en cursiva son palabras inventa. das, para no falsear las respuestas con recuerdos de formulas apiendidas en la escuela.

(N. del T.: las palabras en cursiva son las que figuran en el texto original.)

La sinfonanza de un carore es directamente proporcional a ia $t a n z a, \mathrm{~L}$, del catore, al garone, $\mathrm{G}$, aplicado e inversamente proporcional a la punita, $\mathrm{P}$.

a) Expresar la afirmación mediante una fórmula algebraica.

b) Representar gráficamente la afirmación usando el/los gráficos convenientes.

b) Transposición en gráficos. Para esta pregunta es notable la cantidad de respuestás en blanco ya que más de un tercio no realiza los gráficos y manifiesta explícitamente, en la mayoria de los casos, su propia incapacidad. A esto se agrega que el número de gráficos hechos correctamente, o casi, es muy bajo, excepto para dos escuelas con opción técnico-científica. Analizando los gráficos producidos se observa una marcada tendencia a intercambiar los ejes coordenados, poniendo la variable dependiente (la sinfonanza) en el eje de las abcisas.

Se observa también una fuerte exigencia de graduar los ejes, operación que, evidentemente, es considerada necesaria para construir mediante puntos un gráfico lineal.

Ambos fenómenos pueden ser interpretados como síntomas de una muy escasa lamiliaridad con el instrumento gráfico.

\section{Del lenguaje algebraico al lenguaje verbal}

Para evaluar la habilidad de pasar del lenguaje algebraico al verbal se propusieron dos tests: el primero presenta Ia fórmula que expresa la ley de Coulomb de la fuerza entre dos cargas eléctricas (un ejemplo típico de contexto físico no muy desarrollado como contenido en la escuela secundaria); $\mathrm{el} \mathrm{segundo} \mathrm{alude} \mathrm{a} \mathrm{un} \mathrm{contexto} \mathrm{de} \mathrm{la}$ vida cotidiana (Fig. 2, 3). 
Figura 2

Test 2

La fuerza, $f$, entre dos iones cargados respectivamente con cargas $q y$ $Q$, colocillos a la distancia $R$, se expresa mediante la fórmula:

$$
f=k q Q / R^{2}
$$

donde $k$ es una constante.

Expresar con palabras el significado de la lórmula.

Figura 3

Test 3

La afociación inglesa de amas de casa ha propuesto que el sueldo anual $\$$ dé las amas de casa casadas seá calculado según la sjgujente fórmula:

$$
\mathrm{S}=\boldsymbol{a} \mathrm{nN} / \mathrm{R}
$$

siendo $a$ un factor preestablecido e igual para todas, $n$ el número de componentes de la familia, $N$ el número de las habitaciones de la casa en la que vive la familia y $R$ el valor monetario de los regalos recibidos del marido en un año.

a) Expresa en palabras el significado de la fórmula aumque no re parezca aprepiada.

b) Si la formula no te parece apropiada especifica las tazones, eventualmerte, con ejemplos.

En este último caso, la fórmula propuesta pucde considerarse inadecuada, ya que el sueldo calculado de acuer- do con ésta resulta infinito para un valor cero de los regalos del marido. La capacidad para expresar un juicio de adecuación o no de una expresión algebraica es, obviamente, una característica esencial de un buen dominio del lenguaje algebraico. Esta capacidad no podía ser examinada en el test sobre la ley de Coulomb porque está demasiado relacionado con el conocimiento de la conceptualización física de la fuerza. Se ha considerado que presentar la fórmula en el test número 3 como la propuesta de una imaginaria asociación de amas de casa inglesas estimularía la actitud crítica en relación con la propuesta misma. La relación presentada, con la anomaÍa hacia infinito para $\mathrm{R}$ iendiendo a cero, implica una inadecuación de tipo matemático: la capacidad de darse cuenta de tal inadecuación está puesta a prueba con la segunda pregunta.

Los dos tests fueron propuestos a los mismos estudiantes, de modo que se pudiera reconocer, mediante la confrontación de las respuestas al test 2 y a la primera pregunta del test 3 , la importancia de los conocimientos nocionísticos.

La lectura de una formulación algebraica como la propuesta, en la cual sólo aparece la propocionalidad directa $e$ inversa (el cuadrado de una magnitud en el test 2 y la primera potencia en el test 3 ), puede ser realizada correctamente de dos maneras:

a) Sumariamente: Se explicita el significado de la dependencia funcional correlacionando las variaciones de la variable dependiente con las variaciones de las variables independjentes. Ejemplo: «la fuerza aumenta con el aumento de las cargas $q$ y $Q$ y disminuye con el cuadrado de la distancia entre ellas».

b) Analíticamente: Se explicita el significado de la dependencia funcional efectuando una lectura de tipo

Tabla I

\begin{tabular}{|c|c|c|c|c|c|}
\hline & \multicolumn{4}{|c|}{ Respuestas correctas } & \\
\hline Test & $\begin{array}{c}\text { En base } \\
\text { al } \\
\text { significado }\end{array}$ & $\begin{array}{c}\text { Con } \\
\text { razonamiento } \\
\text { analítico }\end{array}$ & $\begin{array}{l}\text { Significado y } \\
\text { razonamiento } \\
\text { analítico }\end{array}$ & Por lectura & Totales \\
\hline Ley de Cotulomb & 6 & 40 & 16 & 16 & 78 \\
\hline Ama de casa & 14 & 33 & 6 & 25 & 78 \\
\hline $\begin{array}{l}\text { Cohcrencia en los } \\
\text { dos tests }\end{array}$ & 6 & 28 & 2 & 14 & 50 \\
\hline
\end{tabular}

Frecuencia porcentual de las respuestas correctas a las tres cuestioncs, subivididas según la inodalidad de respuesta. 
analítico de la fórmula. Ejemplo: «la fuerza es proporcional al producto de las cargas e inversamente proporcional al cuadrado de la distancia» con o sin el agregado: «con una constante de proporcionalidad K».

Otra modalidad diferente de respuesta, que parece no incluir la comprensión del signifcado, se tiene, en cambio, cuando se da una lectura descriptiva de la fórmula. Ejemplo: «la fuerza es igual a $\mathrm{K}$ por el producto $\mathrm{qQ}$ dividido $\mathrm{R}^{2}$ ».

Las respuestas de los estudiantes, que figuran en la tabla I, muestran que la mayoría de ellos es capaz de responder correctamente a las preguntas usando los diversos criterios, pero que el criterio analítico es el preferido. Se observa también una coherencia razonable entre las respuestas a las dos preguntas.

Las respuestas que se refieren a determinar si la fórmula en el test 3 es adecuada o no se categorizaron como sigue:

- Se usa un criterio numérico con o sin unidad de medida. Ejemplo: «la fórmula no está bien porque, si hay tres personas en dos habitaciones con 100.000 de sueldo y 10.000 de regalos, resulta... que es demasiado bajo"s.

- Se usa un criterio socioeconómico como en el ejemplo: «tienen importancia el tamaño de las habitaciones, la calidad de la decoración, la utilidad de los regalos y la calidad del trabajo».

- Respuestas en las que se detecta un criterio parcialmente emotivo. Ejemplo: «No es justo usar una fórmula porque una ama de casa se ocupa del hogar no por obligación sino por afecto!» «¡No es correcto introducir en el cálculo dej sueldo los regalos del marido!»

- El criterio adoptado es analitico. Se identifican correctamente las inadecuaciones matemáticas de la fórmula en el problema de qué dimensiones atribuir a la constante de proporcionalidad o en la divergencia a infinito para $\mathrm{R}=0$.

Un elevado número de estudiantes (37) no responde a la pregunta. La adopción del criterio socioeconómico es elevada (47). No es despreciable la adopción del criterio numérico (8) y del emotivo (8). El criterio analítico fue

Figura 4

Test para la evaluación de la habilidad de lectura de un gráfico.

Examina atentamente la figura.

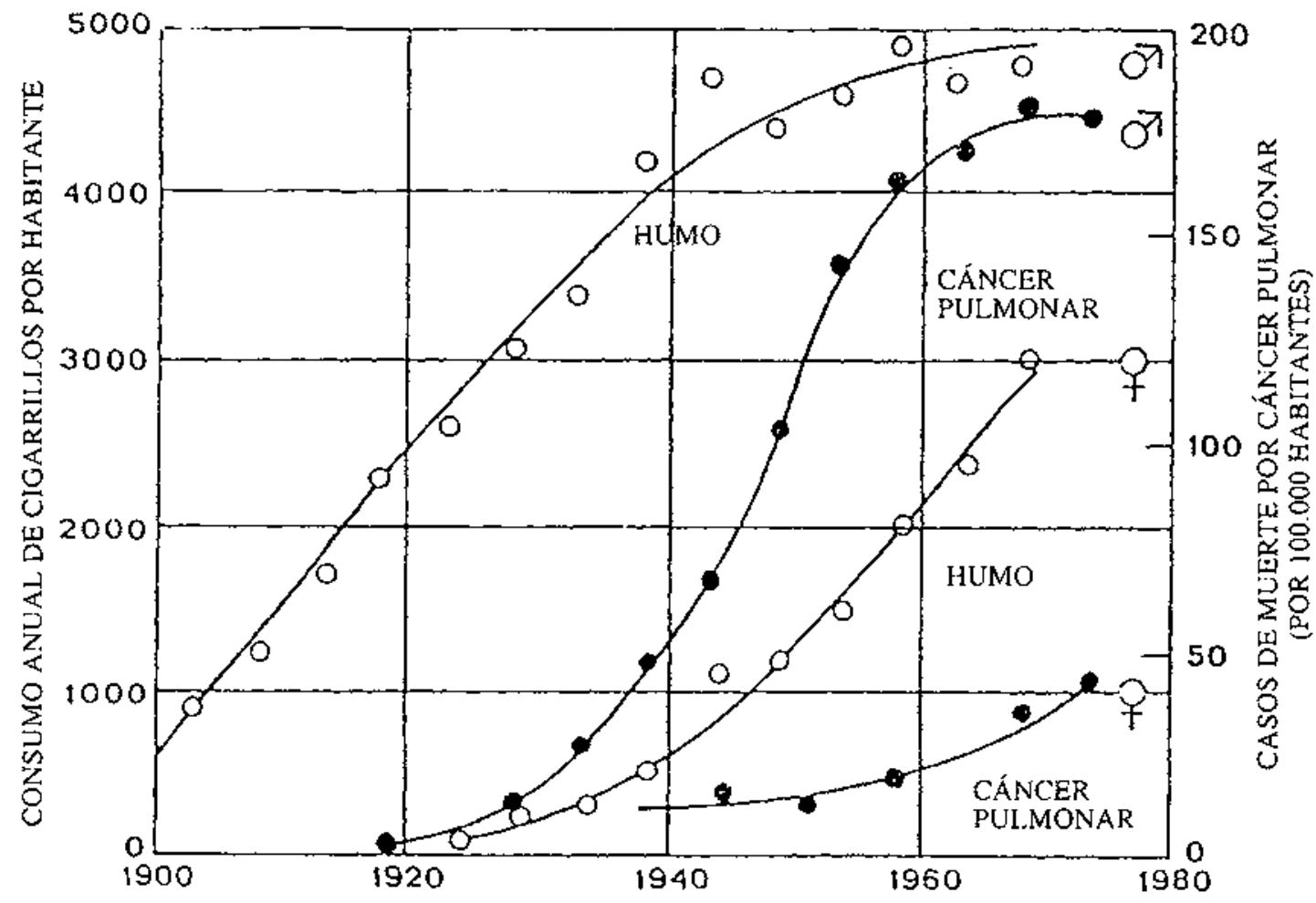

En ha figura se representan los datos relativos al consumo anual de cigarrillos (o) y los casos de cáncer de pulmón sobre 100 goo habitanles ( *), referidos a hombres ( $(O)$ ) y a mujeres ( $(O)$ ) en Inglaterra.

Utilizando exclusivamente los datos ieprescntados en el gráfico, ciasifica las afirmaciones enumeradas en la página siguiente señalando con una cruz las que consideres adecuadas. 
Tabla It

Frecuencias porcentuales de clasificación de las ocho afirmaciones referidas a los fenómenos representados en la figura 4 (clave: $V=$ verdadera en base al texto, $F=$ falsa en base al texto, NA = no autorizada (no se deduce del texto), $\mathrm{P}=$ plausible, ND = no sé decidir, En negrita, las clasificaciones correctas; señaladas con asterisco aquellas preferibles).

\begin{tabular}{|c|c|c|c|c|c|}
\hline & $\mathrm{V}$ & $F$ & $\mathrm{NA}$ & $P$ & ND \\
\hline $\begin{array}{l}\text { 1. La recogida de los datos ha sido hecha } \\
\text { cada veinte años. }\end{array}$ & 62.4 & 28,4 & 0.9 & 8,3 & - \\
\hline $\begin{array}{l}\text { 2. Antes del } 1930 \text { las mujeres inglesas no } \\
\text { morían de cáncer. }\end{array}$ & 56.9 & 20,2 & $16,5^{*}$ & 2,8 & 3.6 \\
\hline $\begin{array}{l}\text { 3. El cáncer pulmonar es provocado } \\
\text { predominantemente por el humo. }\end{array}$ & 35,8 & 1,8 & $20,2^{*}$ & 35,8 & 6,4 \\
\hline $\begin{array}{l}\text { 4. Se puede predecir que en } 1990 \text { no habrà } \\
\text { más diferencia entre los hombres y las } \\
\text { mujeres ingleses en el consumo de cigarrillos. }\end{array}$ & $10,1^{\prime:}$ & 23,9 & 22.0 & $34,9=$ & 9.1 \\
\hline $\begin{array}{l}\text { 5. El consumo de cigarrillos conlleva el mismo } \\
\text { riesgo de enfermarse de cáncer pulmonar tanto para } \\
\text { los hombres como para las mujeres. }\end{array}$ & 31,9 & 45,0 & $10,1^{*}$ & 27,5 & 5,5 \\
\hline $\begin{array}{l}\text { 6. El consumo de cigatrillos por las mujeres } \\
\text { inglesas ha aumentado aproximadamente } \\
\text { cinco veces entre } 1940 \text { y } 1970 \text {. }\end{array}$ & $63,3^{*}$ & 23,9 & 1.8 & 6.4 & 4 \\
\hline $\begin{array}{l}\text { 7. Un hombre que fuma consume mâs } \\
\text { cigarrillos que una mujer fumadora. }\end{array}$ & 46.8 & 10,1 & 25,7 & 8,2 & 9 \\
\hline $\begin{array}{l}\text { 8. En el caso de las mujeres, inciden otros } \\
\text { factores, ademais del humo, en el elevado número } \\
\text { de casos de céncer pulmonar. }\end{array}$ & 2,8 & 5,5 & $54,1^{*}$ & 13,8 & 2,3 \\
\hline
\end{tabular}

adoptado por 20 estudiantes y sólo uno de ellos propone una modifficación de la fórmula.

Del lenguaje gráfico al lenguaje verbal (lectura de un gráfico complejo)

Se pide a los estudiantes que establezcan si ocho afirmaciones inherentes a los fenómenos representados en un gráfico (Fig. 4) son «verdaderas, falsas, no autorizadas o plausibies», indicándoles que hagan referencia, exclusivamente, a los datos representados en el gráfico.

Las afirmaciones (Tabla II) pucden ser clasificadas con relación al hecho de que los datos del gráfico proporcionen (afirmaciones 1, 2, 4 y 6) o no (afirmaciones $3,5,7$ y 8) informaciones para la convalidación o cl rechazo. Las respuestas permiten evaluar la capacidad de lectura «directa» del gráfico, de comparación entre datos internos del gráfico y de elaboración de inferencias.

En la tabla II se presentan las frecuencias porcentuales de las respuestas dadas por 109 estudiantes romanos (de 15 clases correspondientes a 4 opciones distintas de la escuela secundaria superior).
Es evidente que la lectura directa del gráfico presenta dificultades nolables. Por cuanto concierne a las relaciones entre puntos y datos (afirmación 1) menos dc un tercio de las clasificaciones son correctas, en particulatr el significado de la ausencia de datos (afirmación 2) no es tenido en cuenta en los dos tercios de las clasificaciones. La comprensión puntual-extensión y limites ". de las variables en exament se verffica en la mitad de las clasificaciones correctas, en el caso de la afirmación 3 , pero sólo en un cuarto de las clasificaciones correctas en el caso de la afirmación 7.

La comparación entre los datos interiores del gráfico $\mathrm{es,}$ como mucho, tratada correctamente si se opera en el ámbito objetivo de la representación sobre una sola curva (afirmación 6: dos tercios de respuestas correctas), resultando más problemático en las extrapolaciones (afirmación 4).

La lectura inferencial parece de buen nivel (afirmaciones 5 y 8 ), ya que más de la mitad de las clasificaciones son aceptables. No resulta fácil establecer, sin embargo, si las elecciones hechas han sido precedidas, como es necesario y solicitado, de un análisis del gráfico, o $\mathrm{si}$ 
fueron hechas exclusivamente a partir de convicciones anteriores, y coinciden casualmente con la valoración correcta. Esta última alternativa parecería avalada por la evidencia de un defecto general de atención y de implicación en un análisis crítico (aun en la lectura de las consignas): una situación que conlleva el riesgo del predominio -en la construcción del juicio-de las opiniones personales y de sentido común, sobre los datos objetivos (véase en especial la clasificación de la afirmación 7).

En síntesis, se puede afirmar que la lectura de un gráfico cartesiano no se afronta normalmente con rigor metodológico y resulta escasamente productiva para una gran parte de los estudiantes, independientemente del tipo de escuela al que asisten.

\section{Lectura de datos experimentales}

Se trata de una habilidad compleja que incide en la adquisición, interpretación y organización en un orden lógico de las informaciones disponibles, y en la discriminación por coherencia y congruencia entre diversas deducciones posibles.

Fue presentado un experimento histórico planificado y realizado para verificar la hipótesis de la generación espontănea de microorganismos, a partir de sustancias orgánicas, en caldo de carne (Fig. 5). Se enumeran ocho afirmaciones (Tabla III) cuya función es interpretar las observaciones realizadas: se solicita valorar si las afirmacines son deducibles o no a partir de las observaciones. Al pedirles que indiquen qué observaciones se «tuvieron en cuenta para decidir», se tiende fundamentalmente a centrar la atención sobre las consignas y sobre los datos.

Cabe destacar que:

- en cinco casos el porcentaje de elección no se aparta del $50 \%$ que señala la casualidad de la elección misma;

- las observaciones a las que se hace referencia para hacer la elección no se indican sistemáticamente (en el $50 \%$ de los casos);

- no se formulan comentarios significativos.

La capacidad de dominar los instrumentos y procedimientos experimentales se pone a prueba a tres niveles:

- El primer nivel está representado por las afirmaciones 5 y 6 en las que se mencionan explícitamente dos variables -temperatura y tiempo de incubación, respectiva-

Figura 5

Test para la evaluación de las habilidades de lectura de datos experimcntales.

Teniendo en cuenta la obscrvación de que muchos organismos vivientes se encuentran sobre todo cerca o en el interior de sustancias orgánicas en descomposición (por cjemplo, moscas de la fruta, gusanos y mufa en el queso, microorganismos en caldos e infusiones) algunos cientificos han formulado la hipótesis de que estos organismos se originan espontáneamente a pastir de esas sustancias.

Para verificar experimentalmente tal hipótesis se puede pensar en tratar de modos diferentes cuairo series de probetas contenicndo caldo de carne turbio por la presencia de microorganismos vivos y lucgo compararlas, haciendo un lotal de ocho grupos de observaciones.

En la tabla siguiente se muestran los tipos de tratamiento (líneas) y se indica el aspecto que presenta el caldo en las probelas, después de una hora y de diez dias de haber realizado el tratamiento, respectivamente (columnas). Las ocho casillas numeradas corresponden a las observaciones.

\begin{tabular}{|c|c|c|c|c|c|}
\hline & & \multicolumn{4}{|c|}{ Aspecto del caldo despucs de: } \\
\hline & & & 1 hora & & 10 dias \\
\hline \multirow{4}{*}{ 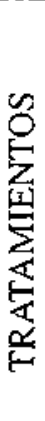 } & $\begin{array}{l}\text { Probetas cubicrrtas con una gasa: } \\
\text { calentamiento durante } 40 \text { min. a } 60^{\circ} \mathrm{C}\end{array}$ & 1 & curbiso & 2 & turbio \\
\hline & $\begin{array}{l}\text { Probetas cubierlas con una gasa: } \\
\text { calentamiento durante } 40 \text { min. a } 100 \text { "C }\end{array}$ & 3 & claro & 4 & curbio \\
\hline & $\begin{array}{l}\text { Probetas selladas con cera: } \\
\text { catentamiento durante } 40 \mathrm{~min} \text {. a } 60^{\circ} \mathrm{C}\end{array}$ & 5 & turbio & 6 & iurbio \\
\hline & $\begin{array}{l}\text { Probetas selladas con cera: } \\
\text { calentamiento durante } 40 \text { min. a } 100 \text { 'C }\end{array}$ & 7 & claro & 8 & claro \\
\hline
\end{tabular}

Para interpretar estos datos se proponen las afirmaciones abajo enumeradas.

Indica para cada una de ellas si la consideras demostrada de acuerdo con los datos (señala con una cruz ła casilla correspondiente a tu evaluación). Especifica además -en la coltumna correspondiente- el número de orden de las observaciones que se han tenido en cuenta para decidir. 
Tabla HII

Frecuencias porcentuales de clasificación correcta de nueve afirmaciones que interpretan el experimento prescntado en la figura 5 , conjuntamente o respecto a observaciones aisladas (clave: $\mathrm{D}=$ afirmación demostrada, $\mathrm{N}=$ afirmación no demostrada).

\begin{tabular}{|c|c|c|}
\hline & $\mathrm{D}$ & $\mathrm{N}$ \\
\hline 1. La generación espontánea es posible. & & $5 i, 0$ \\
\hline 2. La generación espontánea es imposible. & & 42,6 \\
\hline 3. El oxígeno no es necesario para la vida de los organismos presentes en el caldo. & 54,9 & \\
\hline 4. La gasa permite que entre aire en la probeta. & 84,3 & \\
\hline 5. Los organismos presentes en el caldo no mueren a la temperatura de $60^{\circ} \mathrm{C}$. & 89,6 & \\
\hline 6. Para que se formen microorganismos en el caldo son suficientes 10 dias. & 70,8 & \\
\hline 7. Para obtener resultados significativos es necesario calentar durante 40 minutos. & & 58,1 \\
\hline 8. Los organismos que cnturbian cl caldo no se originan a partir del caldo. & 52,4 & \\
\hline 9. Los microorganismos se generan ch el caido como los gusanos en el queso. & & 68,2 \\
\hline
\end{tabular}

mente-relacionadas (Fig. 5) con el parámetro turbidez (o presencia de organismos vivos) del sistema que se examina. Se registran buenos resultados.

- En el segundo nivel se incluyen las afirmaciones 3 y 7 que requieren una ulterior elaboración lógica: la primera, una inferencia (probeta sellada = el aire no se renueva; aire = oxígeno); la segunda, un evaluación de la incidencia de una variable fija (el tiempo de calentamiento). La calidad de los resultados es baja.

- En el tercer nivel se coloca la interpretación global: la correcta, aparece ampliamente compartida si se mantiene la conexión con los «hechos» experimentales (afirmación 4), mientras que se registra una gran desorientación si se aleja de los mismos (afirmación 8).

También en lo que concierne a la generalización de las conclusiones, los resultados son mejores cuando hay una referencia a lo "concreto» (afirmación 9) que cuando hay una valoración «abstracta» (afirmaciones 1 y 2 ). Es necesario aclarar que en el primer caso la elección clasificatoria se debe relacionar con el conocimiento de las diversas peculiaridades de los microorganismos y de los gusanos (reproducción mediante esporas y huevos, respectivamente; difusión en el aire de las esporas y deposición de los huevos por el insecto). La eleccion, por lo santo, podría no ser atribuible ni a cuestiones de orden experimental, ni a las "modalidades de generación». Se puede en todo caso pensar que no consideran importante la exigencia de rigor lógico: téngase en cuenta que cerca de $10 \%$ de los estudiantes considera demostradas tanto la primera como la segunda afirmaciones (muchos, correctamente, tas consideran no demostradas).

\section{DISCUSIÓN}

La conexión entre dos niveles escolares consecutivos no puede prescindir, en principio, de una definición explícita y de un respeto riguroso de las respectivas obligaciones đe formación. En la práctica, por to tanto, los docentes universitarios deberían tener en cuenta los programas didácticós desarrollados en la escuela secundaria superior y los niveles de habilidades cognitivas y de estudio en ella adquiridos, y deberían poder contar con ellos, poniéndolos en confrontación sistemáticamente con los conocimientos y habilidades necesarios para iniciar de modo eficaz el itinerario didáctico universitario.

Esta confrontación no se ha hecho nunca en la escuela italiana: sería, en todo caso, dificultoso considerando el gran número de opciones existentes en la escuela secundaria superior, la variedad de los programas didácticos dentro del ámbito de una misma opción (variedad potenciada por las inciativas de experimentación que promueven la innovación didáctica y curricular), la escasa práctica de métodos para evaluar la eficacia de las intervenciones educativas y la casi total liberación del acceso a las facultades universitarias.

Parece inevitable, por todo ello, que los estudiantes inscritos en el primer año de una carrera universitaria dispongan de conocimientos, competencias y experiencias extremadamente heterogéneas; y parece, en consecuencia, razonable que los docentes universitarios limiten sus expectativas a ese núcleo de habilidades de base que deberían adquirir en el curso de los estudios, casi independientemente del tipo y de la especificidad de los mismos. 
Ahora bien, Jos datos recogidos en cinco escuelas romanas evidencian un nivel de rendimiento bajo en relación con el mínimo necesario para un curso de ciencias universitario (tal como son concebidos y organizados actualmente). Los conocimientos requeridos implican, en gencral, una actitud analítica y una buena disposición a la formalización y, especialmente, al uso, comprensión y producción de lenguajes algebraico y gráfico, así como a la lectura de datos experimentales.

I a fisonomf́a del estudiante que se inscribe a la universidad, valorada respecto del proceso de transformación en «especialista», es muy parecida a la del «hombre o mujer común» por muchas razones: prefiere afrontar pragmáticamente problemas concretos antes que empeñarse en la realización de actividades de formalización (véase los criterios considerados para la evaluación de la adecuación de la fórmula en los tests 2 y 3 ). Es posible que el estudiante conciba como problema concreto la aplicación de una regla o de una fórmula para la solución de un «cjercicio», especialmente si el ámbito fenomenológico y el léxico utilizados en el ejercicio son los propios de un sector disciplinario determinado. Se trata de una situación en la que la eexperiencia» personal del estudiante (conocimientos y comportamientos) coincide con lo que ha aprendido en la escuela.

Esta última consideración introduce una segunda peculiaridad del estudiante que lo acerca al «hombre común»: el estudiante recurre selectivamente a su experiencia personal, aun disponiendo de datos más generales $y$ en buena medida validiados, cuando se le pide que se reficra exclusivamente a esos datos. La presencia, en el gráfico de la figura 4 , de los datos que permiten validar o rechazar un afirmación en particular (tabla lI, afirmaciones 3 y 7 ), no representan - para la mayoría de los estudiantes- un discriminante para la clasificación correcta de la afirmación. Essta actitud se manifiesta claramente también en relación con el test de la figura 5 .

$\Lambda$ la exigencia de concreción manifestada por los estudiantes se agrega la predilección por los "hechos", que se traduce en mejores rendimientos allí donde las consignas implican un grado medio-bajo de inferencia, extrapolación y generalización (véase la tipología de los criterios en el test núm. 3, la afirmación 4 en la tabla II y las afirmaciones $1,2,3$ y 8 en la tabla IIl).

Respecto a las habilidades básicas («leer, escribir y hacer cálculos»), resulta suficientemente evidente y argumentado el escaso dominio de los instrumentos de lectura, que orientan la atención y el interes, que garan-

\section{REFERENCIAS BIBILIOGRÁFICAS}

BANDIERA, M., 1990. Un'indagine sulle abilità di studio, Atti dei seminari di Didattica delle Scienze biologiche, pp. 57.73 (Cirda: Turín).

BRONOWSKY, J., 1980. Le origini della conoscenza e dell'immaginazione. Roma.

CHI, M.T.H., FELTOVICH, P.J. y GLASER, R., 1981. tizan una «lectura puntual» eficaz (identificación de todas las informaciones presentes en el texto) y la transforman en «lectura inteligente» (categorización y asimilación de las informaciones para una comprensión total del texto). Es necesario, por otra parte, reconocer que la escuela italiana, comprendida la universidad, en general no promueve la lectura ni se ocupa de ello en el plano metodológico y, en particular, no la ejercita en relación con los lenguajes no-verbales propios de la comunicación científica. Resulta entonces favorecida la práctica de la memorización pasiva y de la aplicación automática de reglas y fórmulas, así como las formas de espontaneidad y aproximación (véase frecuencia y tipos de lectura de las fórmulas en la tabla I y de lectura de gráficos en la tabla II).

También la escritura en los lenguajes algebraico y gráfico (Test núm. 1) evidencia grandes carencias en cuanto a su planifícación y práctica. Así mismo, cl hacer cálculos muestra las carencias en las capacidades de formular claboraciones relacionadas con los datos, conceptualmente significativas y rigurosamente lógicas (Tabla III).

Dado que no han sido encontradas diferencias notables entre los resultados obtenidos de estudiantes de diversas opciones escolares ( $s i$ se excluyen aquellas atribuibles a la heterogeneidad de Ios niveles económicos) y, sobre todo, considerando cl hecho de que a cada curso universitario pueden inscribirse estudiantes provenientes de todas las opciones de la escuela, se debc tomar conciencia de la existencia de una sustancial discontinuidad entre la escuela secundaria superior y la universidad, discontinuidad que deberá ser solucionada con la reforma que se lleva a cabo actualmente, pero que, en la actualidad es causa probablemente de las grandes dificultades de los estudiantes universitarios (bajos niveles de presentismo, altos porcentajes de suspendidos y de abandonos).

Es oportuno subrayar, por último, que los datos y consideraciones presentados no tienen únicamente finalidades de documentación, sino que pueden orientar las innovaciones de los temas y de las metodologias de la enseñanza preuniversitaria, pueden promover la institución de «lineamientos propedéuticos» por parte de las facultades, y pueden prefigurar instrumentos de diagnóstico que ayuden a los neodiplomados a elegir el tipo de cstudios universitarios que puedan afrontar con menos dificultades y con la máxima probabilidad de éxito.

\section{NOTA}

Este articulo ha sido traducido del italiano por Silvia Gamero.

Categorization and representation of physics problems by experts and novices, Cognitive Science, 5, pp. 121-152.

HACKLING, M.W. y LAWRENCE, J.A., 1988. Expert and novice solutions of genetic pedigrees problems, Journal of Research in Science Teaching, 25, pp. 53!-546.

PFUNDT, H. y DUIT, R., 1990. Students' Alternative frameworks and Science Education, A bibliography IPN-Kiel. 